\title{
Recent Advances in 2D and 3D TOF SIMS Analysis of Organic and Inorganic Surfaces
}

Felix Kollmer ${ }^{1}$, Alexander Pirkl $^{1}$, Sven Kayser ${ }^{1}$, Henrik Arlinghaus ${ }^{1}$, Rudolf Moellers ${ }^{1}$, Nathan Havercroft $^{2}$ and Ewald Niehuis ${ }^{1}$

${ }^{1}$ IONTOF GmbH, Münster, Nordrhein-Westfalen, Germany, ${ }^{2}$ IONTOF USA, Inc., Chestnut Ridge, New York, United States

Time-of-flight secondary ion mass spectrometry (TOF-SIMS) is known to be an extremely surface sensitive analytical technique. It provides detailed elemental and molecular information about surfaces, thin layers, interfaces, and full three-dimensional analysis of the sample.

In our contribution we will provide an overview on recent advances in TOF-SIMS instrumentation and data evaluation that are extending existing applications or even make new applications accessible. Among the discussed improvements are key values that are inherent for a dedicated TOF-SIMS instrument as mass resolution, lateral resolution and the ability to obtain both in parallel, as well as improvements that are based on the integration of additional analytical components or due to the combination with complementary techniques.

The latest technology step now pushes the standard lateral resolution of dedicated TOF-SIMS instruments to the sub $50 \mathrm{~nm}$ region. Best achieved values are even in the $20 \mathrm{~nm}$ range and therefore not far away from the physical limit given by the size of the sputter cascade [1]. Since the achievable lateral resolution results from a convolution of the beam spot size and the size of the sputter cascade at the surface, even a further decrease in the spot size of the primary ion beam, leads only to minor improvement of the achievable lateral resolution [2].

While new ion sources expanded the usability of SIMS instruments, SIMS analyzers lacked the required mass resolution, mass accuracy and MS/MS capabilities, required for the thorough investigation of molecular materials. One major problem is based on the enormous number of molecules in the mass range of interest (see figure 1).

An improtant step in order to reduce the number of interpretation possibilities is to improve the mass resolution and the mass accuracy. State-of-the-art TOF-SIMS instruments now are reaching a mass resolution $(\mathrm{m} / \Delta \mathrm{m})$ in the range of 30.000 and a mass accuracy in the range of several ppm. This improves the analytical possibilities compared to the previous instrument generation and reduces the complexity of data evaluation and interpretation.

However, the number of remaining possibilities for a given peak originating from molecular surfaces and especially from biological samples can be still too high for an unambiguous peak assignment. In these cases, MS/MS capabilities are required in order to further increase identification possibilities. As the MS/MS fragment ion spectrum is almost independent of the ion generation of the precursor, one can benefit from the large number of reference spectra collected in various libraries. In this respect it is also of high importance to generate the MS/MS spectra at high mass resolution and high mass accuracy.

We will discuss an MS/MS approach that is fully integrated into a TOF analyzer. The benefit of this approach is that it can easily be integrated in the given analysis scheme and data format. Moreover, for certain energy classes it is beneficial that ions are generated in a high energy collision process. The applied 90-degree momentum deflection system enables a high transmission and a high mass resolution precursor selection.

While the discussed performance represents the limit attainable with recent TOF-SIMS instrumentation, further improvement is achieved by combining an OrbitrapTM-based mass analyzer (Q ExactiveTM HF, Thermo Fisher ScientificTM) with a high-end TOF-SIMS system (Hybrid SIMS) [4]. The instrument 
provides highest mass resolution ( $>240,000)$ and highest mass accuracy $(<1 \mathrm{ppm})$ with high lateral resolution cluster SIMS imaging. We will present data acquired on a Hybrid SIMS instrument demonstrating fast and robust identification of main constituents in unknown samples.

The high complexity of a molecular secondary ion mass spectrum and the high number of secondary ion peaks not only hampers peak identification but also makes it challenging to identify the peaks that are relevant for the given analytical problem. We will present a new concept of multiplexing SIMS acquisitions and demonstrate its application to selected samples.

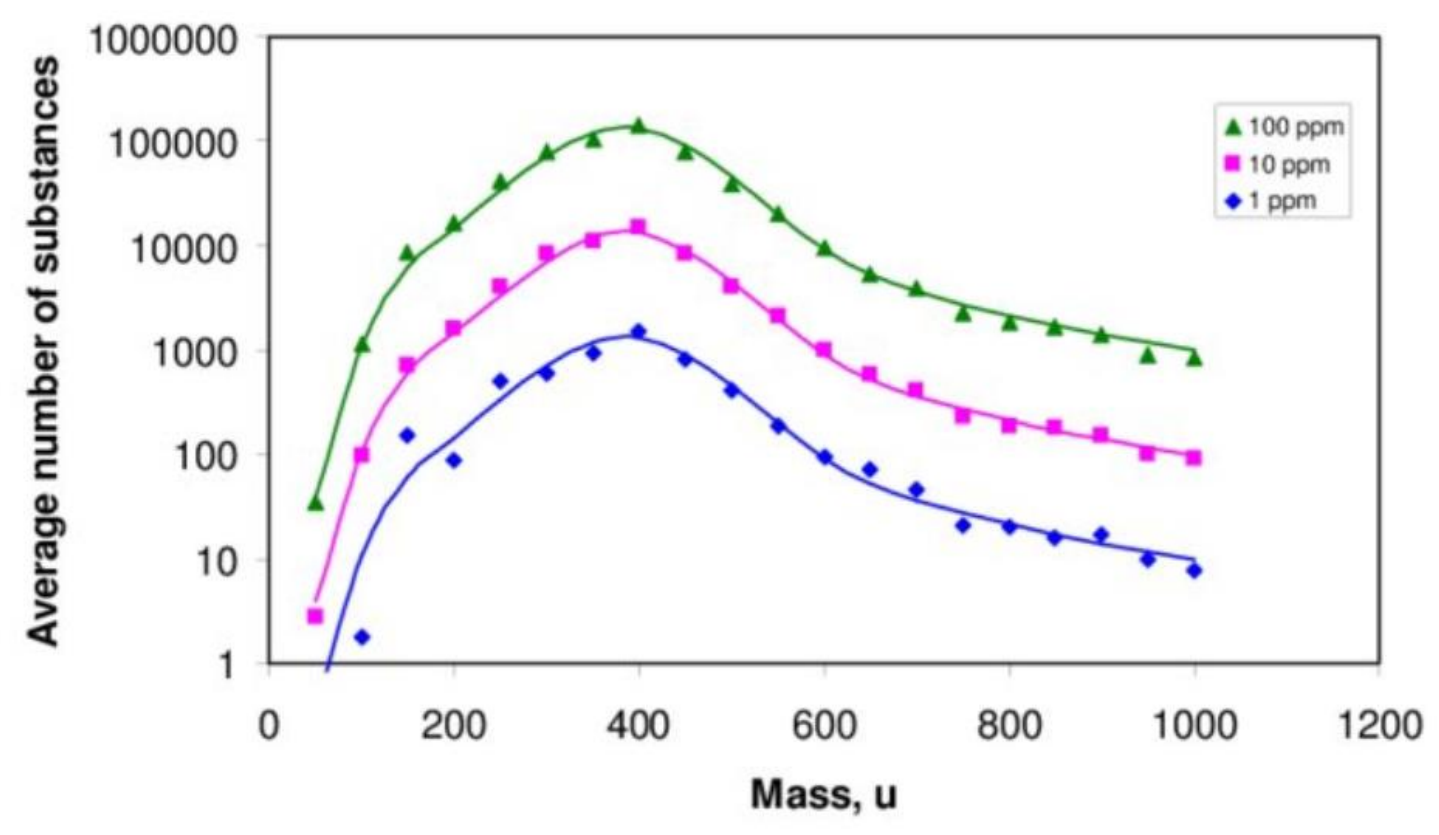

Figure 1. Average number of molecules in PubChem data base [3]

\section{References}

[1] Kollmer, F., Paul, W., Krehl, M. and Niehuis, E. (2013), Ultra high spatial resolution SIMS with cluster ions - approaching the physical limits. Surf. Interface Anal., 45: 312-314. doi:10.1002/sia.5093

[2] Sijbrandij, S., Lombardi, A., Sireuil, A., Khanom, F., Lewis, B., Guillermier, C., Notte, J. (2019). NanoFab SIMS: High Spatial Resolution Imaging and Analysis Using Inert-Gas Ion Beams. Microscopy Today, 27(3), 22-27. doi:10.1017/S1551929519000440

[3] I. S. Gilmore, SIMS of organics-Advances in 2D and 3D imaging and future outlook, Journal of Vacuum Science \& Technology A 31, 050819 (2013); https://doi.org/10.1116/1.4816935

[4] Passarelli, et al. Nature Methods volume 14, pp 1175-1183 (2017) 